\title{
Bloqueio do nervo alveolar mandibular com ropivacaína a 0,5 \% em gatos
}

\author{
Bockage of the jaw's alveolar nerve with $0.5 \%$ ropivacaine in cats
}

\author{
Vanessa Martins Fayad Milken ${ }^{1}$ Patrícia Maria Coletto Freitas ${ }^{2}$ Duvaldo Eurides ${ }^{3}$ Frederico Ozanam \\ Carneiro e Silva ${ }^{3}$ Francisco Cláudio Dantas Mota ${ }^{4}$ Elisete de Araújo Naves ${ }^{5}$ Renata Junqueira Rezende ${ }^{6}$ \\ Leandro Arévalo Prieto ${ }^{7}$ Mariana da Silva Melo ${ }^{7}$ Michele Rodrigues Goulart ${ }^{8}$
}

\section{RESUMO}

Objetivou-se, com este experimento, avaliar a ação da ropivacaína a $0,5 \%$ no bloqueio do nervo alveolar mandibular de gatos. Vinte gatos adultos, sem raça definida, machos ou fêmeas, receberam clorpromazina $\left(1,0 \mathrm{mg} \mathrm{kg}^{-1}, \mathrm{VO}\right)$ e propofol (3,0mg kg-1, IV). Ropivacaína a 0,5\% foi administrada com uma agulha $13 \times 3,8$ em forma de “ $L$ ", inserida no ângulo da mandíbula direita, aproximadamente $1,0 \mathrm{~cm}$ rostral ao processo angular e $0,5 \mathrm{~cm}$ dorsal à superfície medial do ramo da mandíbula, a fim de depositá-la próximo ao nervo alveolar mandibular, no forame mandibular. As freqüências cardíaca e respiratória foram mensuradas antes da administração da clorpromazina, 20 minutos após administração desta (T0), 20 minutos após o bloqueio do nervo alveolar mandibular com ropivacaína (T20) e, em intervalos de 20 minutos, até a volta da sensibilidade na região anestesiada. Observou-se o período de latência e a duração da anestesia por meio do pinçamento da pele e gengiva da região lateral direita da mandíbula. Encontrou-se início da anestesia após 22 minutos, com duração de 164,25 minutos. Os parâmetros de freqüência cardíaca e freqüencia respiratória tiveram alterações, porém sem significado clínico para a espécie. A ropivacaína a $0,5 \%$ anestesia a região dos dentes pré-molares, molares, caninos, incisivos, pele e mucosa oral e lábio inferior, sem causar efeitos colaterais.

Palavras-chave: gato, anestesia, ropivacaína, nervo alveolar mandibular.

\begin{abstract}
This study intended to evaluate the $0.5 \%$ ropivacaine action on the alveolar mandibular nerve block in cats. Twenty adult cats, non-defined breed, male or female, received chlorpromazine (1.0 mg kg-1 VO) and propofol (3,0 $\mathrm{mg} / \mathrm{kg} \mathrm{IV}$ ). Ropivacaine at $0.5 \%$ was administrated with an " $L$ " 13x3,8 needle, inserted in the angle of the right mandible, close to $1.0 \mathrm{~cm}$ rostral to the angular process and $0.5 \mathrm{~cm}$ dorsal to the medial surface of the mandible branch, intending to deposit close to the alveolar mandibular nerve, at the mandibular forame. The heart and respiratory rates were measured before the chlorpromazine administration, 20 minutes after its administration (T0), 20 minutes after the alveolar mandibular nerve block with ropivacaine (T20) and in 20 minutes intervals till the sensitivity recovery at the anesthetized region. The latency period and the anesthesia duration were observed through the skin and gingival cross-clamping of the mandible right-lateral region. The anesthesia beginning was found 22 minutes after, with 164.25 minutes duration. The heart rate parameters and respiratory rate had alteration, but without clinical significance to the specie. The $0.5 \%$ ropivacaine anesthesia at the region of the premolars, molars, canines, incisors, skin and oral mucosae and bottom lip, without causing collateral effects.
\end{abstract}

Key words: cat, anesthesia, ropivacaine, alveolar mandibular nerve.

${ }^{1}$ Curso de doutorado em Diagnóstico por Imagem, Universidade Estadual Paulista (UNESP), Botucatu Av. Ana Godoy de Souza, 764, B, Santa Mônica, 38408-290, Uberlândia, MG, Brasil. Email: vanessafayad@yahoo.com.br

${ }^{2}$ Curso de doutorado em Cirurgia, UNESP, Jaboticabal. Prof. de Cirurgia e Anestesiologia Veterinária, Centro de Ciências Agrárias (CCA), Universidade Federal do Espírito Santo (UFES).

${ }^{3}$ Faculdade de Medicina Veterinária (FAMEV), Universidade Federal de Uberlândia (UFU). Uberlândia, MG, Brasil.

${ }^{4}$ Curso de doutorado em Ortopedia, Universidade Federal de São Paulo (UNIFESP), Ribeirão Preto, SP, Brasil.

${ }^{5} U F U$, Uberlândia, MG, Brasil.

${ }^{6}$ Curso de doutorado em Parasitologia, UFU, Uberlândia, MG, Brasil.

${ }^{7}$ Curso de mestrado em Clínica e Cirurgia, UFU, Uberlândia, MG, Brasil.

${ }^{8}$ Médica Veterinária autônoma. 


\section{INTRODUÇÃO}

A anestesia regional surgiu em pequenos animais como uma alternativa para analgesia durante procedimentos cirúrgicos, possibilitando um período anestésico mais estável (GROSS et al., 1997). A aplicação de anestésicos locais antes da produção do trauma cirúrgico limita ou minimiza a reação tissular inflamatória e diminuiu a sensibilidade central à dor, proporcionando ao paciente maior conforto pós-operatório (CEDIEL et al., 1999). A anestesia local oferece vantagens como baixa toxicidade, baixo custo, redução das doses dos demais fármacos utilizados e recuperação mais rápida com analgesia residual. Dentre as desvantagens, podem-se citar o risco de toxicidade, analgesia insuficiente para determinados procedimentos quando utilizada sem associações, a necessidade de precisão na deposição do anestésico para bloqueio de determinados nervos, e ineficácia moderada em regiões com grande depósito de gordura, ossos, cartilagem, fáscias musculares, tendões e outros tecidos conjuntivos (FANTONI \& CORTOPASSI, 2002).

Procedimentos odontológicos em felinos tornaram-se rotina na clínica-cirúrgica de pequenos animais como restaurações dentais e exodontia. Esses tratamentos requerem analgesia no pré e pós-operatório. Os analgésicos comumente usados para estes procedimentos incluem opióides, agonista (-2 adrenérgicos e antiinflamatórios não-esteroidais, que se administrados parenteralmente, podem causar efeitos indesejáveis em gatos (GROSS et al., 2000). Assim, a anestesia regional é uma alternativa como adjuvante aos fármacos sistêmicos para controle da dor durante e após procedimentos dentais em gatos, uma vez que causam efeitos adversos mínimos (GROSS et al., 1997).

O nervo alveolar mandibular é um nervo sensitivo, ramo do nervo mandibular, que penetra no forame mandibular(GODINHO \& GETTY, 1986). Dentro do canal mandibular divide-se em ramos caudais, médios e rostrais, sendo que estes ramos inervam os dentes molares, pré-molares, caninos e incisivos e as gengivas correspondentes, respectivamente (SCHALLER, 1999). Antes de penetrar no canal mandibular, o nervo alveolar inferior emite o nervo milohióide, sendo que este nervo tem característica motora quando inerva o músculo milo-hióideo e, sensorial, quando inerva a pele entre as mandíbulas (EVANS \& LAHUNTA, 1994). Ainda na extremidade rostral do canal mandibular, o nervo alveolar mandibular dividese em várias ramificações que deixam o forame mentoniano como nervos mentonianos que suprem a pele da região mentoniana, inervando queixo e lábio inferior(GODINHO\& GETTY, 1986; SCHALLER, 1999).
Os bloqueios regionais compreendem a deposição do anestésico local nas imediações de um nervo ou grupo de nervos que formam um plexo, causando dessensibilização de área extensa, o que normalmente não seria factível com as anestesias infiltrativas ou tópicas (FANTONI \& CORTOPASSI, 2002). Podem-se usar as seguintes técnicas para a dessensibilização de nervos da cabeça: anestesia do nervo infra-orbital, maxilar, oftálmico, mentoniano, mandibuloalveolar (MUIR III et al., 2001).

A técnica descrita por MUIR III et al. (2001) para anestesia dos dentes molares, pré-molares, caninos, incisivos, pele e mucosa da bochecha e lábio inferior de pequenos animais, é o bloqueio do nervo alveolar inferior. Para tanto, deve-se inserir uma agulha $25 \times 7$ ou $25 \times 5$ no ângulo inferior da mandíbula, aproximadamente $1,5 \mathrm{~cm}$ rostralmente ao processo angular e 1,5cm dorsalmente contra a superfície medial do ramo da mandíbula, em direção ao bordo palpável do forame mandibular. Em estudo com gatos, a referência utilizada por GROSS et al. (2000) foi aproximadamente $1,0 \mathrm{~cm}$ rostral ao processo angular e $0,5 \mathrm{~cm}$ pela superfície medial do ramo da mandíbula.

O procedimento para anestesia do nervo alveolar inferior pode ocasionar danos aos nervos periféricos, sendo que o mecanismo exato para isto é desconhecido, assim como sua prevenção e tratamento. As técnicas convencionais de bloqueios regionais estão associadas a riscos e complicações, como problemas neurológicos e vasculares, injeções intravasculares e problemas musculares. Entretanto, as neuroparalisias em pacientes não são freqüentes quando utilizada esta técnica de anestesia (GIULIANI et al., 2001). Uma incidência de 2,9 a 22\% de aspiração de sangue foi descrita por TAKASUGI et al. (2000) ao inserir a agulha para anestesiar, no homem, o nervo alveolar inferior. Contudo, POGREL \& TAMBY (2000) descreveram uma recuperação espontânea de injúrias causadas por bloqueios regionais após oito semanas de pós-operatório em 85 a $94 \%$ dos casos. AFSAR et al. (1998) observaram falha de 29 a 35\% no bloqueio do nervo alveolar inferior utilizando a fossa pteriogomandibular no homem.

Diversos fármacos foram utilizados para anestesia do nervo alveolar inferior, como a cloroprocaína, em cães (GROSS et al., 1997) e gatos (GROSS et al., 2000), prilocaína (PROGREL \& THAMBY, 2000) e lidocaína (TAKASUGI et al., 2000) no homem.

A ropivacaína é um anestésico local do tipo amida, monoidrato do sal hidroclorídrico do 1-pipecaloxilidídeas, sendo preparado como Senantiômero. Estudos clínicos têm demonstrado maior margem de segurança no uso deste agente para execução de bloqueios regionais que demandam grandes quantidades de anestésico local, devido ao 
distanciamento entre as suas doses terapêutica e tóxica, sem prejuízo da eficácia (DELFINO et al., 1999; DELFINO et al., 2000). Doses e concentrações baixas produzem analgesia confiável com bloqueio motor mínimo e não-progressivo. Na dose máxima recomendada, é mais eficaz que a bupivacaína. Possui longa ação, similar a bupivacaína, porém com menores efeitos tóxicos ao sistema nervoso central (FANTONI \& CORTOPASSI, 2002). Experimentações in vitro e in vivo têm demonstrado ser a ropivacaína menos neurotóxica e cardiotóxica que a bupivacaína, devido à sua forma levógira de amino-amida, possuindo assim um efeito vasoconstritor intrínseco, assegurando menor absorção a partir do local de injeção, com redução do seu nível plasmático (DELFINO et al., 1999; DELFINO et al., 2000). Liga-se 94\% às proteínas plasmáticas e possui alta lipossolubilidade (RAMOS et al., 2000).

A dose da ropivacaína para pequenos animais varia de 0,5 a 2,0 $\mathrm{mg} \mathrm{kg}^{-1}$ (MUIR III et al., 2001). Em ratos e cães, altas concentrações de ropivacaína causam redução no fluxo sangüíneo medular. No entanto, o mesmo não acontece quando doses clínicas são empregadas, acarretando apenas mínimas e transitórias modificações neste fluxo (DELFINO et al., 1999; DELFINO et al., 2000). Observaram-se repercussões neurológicas reversíveis relacionadas à vasoconstrição por isquemia medular, obtidas em animais de experimentação com o emprego de altas doses de ropivacaína (DELFINO et al., 1999). Toxicidade levando à perda da consciência e convulsões tônicoclônicas, com alterações da pressão arterial, freqüência e ritmo cardíaco foram relatados por MARANHÃO et al. (2000) ao injetar acidentalmente ropivacaína intravascularmente no homem.

Objetivou-se, neste experimento, avaliar a ropivacaína a $0,5 \%$ em bloqueio do nervo alveolar mandibular de gatos.

\section{MATERIAL E MÉTODOS}

Para determinação do local a ser realizado o bloqueio anestésico, dissecou-se a região mandibular de três peças anatômicas de gatos, para definir com auxílio de uma régua, a distância entre o forame mandibular e o processo angular da mandíbula e entre este e a borda ventral da mandíbula.

Para a realização do bloqueio do nervo alveolar mandibular foram utilizados 20 gatos adultos, sem raça definida, de ambos os sexos, com peso variando entre 2,0 e 3,0kg. Após jejum completo, realizou-se tricotomia e anti-sepsia na face direita da mandíbula.
Como medicação pré-anestésica, administrou-se clorpromazina na dose de $1,0 \mathrm{mg} \mathrm{kg}^{-1}$, VO e após 20 minutos, aplicou-se propofol na dose de 3,0 $\mathrm{mg} \mathrm{kg}^{-1}$, IV. Em seguida, administrou-se ropivacaína a $0,5 \%$ na dose de $1,0 \mathrm{mg} \mathrm{kg}^{-1}$, com uma agulha $13 \times 3,8$ modificada para a forma de "L", inserida na face medial da mandíbula direita, aproximadamente $1,0 \mathrm{~cm}$ rostral ao processo angular e $0,5 \mathrm{~cm}$ dorsal à borda ventral da mandíbula, a fim de depositar o anestésico próximo à entrada do nervo alveolar mandibular no forame mandibular.

Após a aplicação da ropivacaína, avaliouse o tempo de latência sensitiva e a duração do bloqueio por meio do pinçamento da pele e gengiva da região lateral direita à mandíbula, utilizando como controle o lado esquerdo que não sofreu o procedimento anestésico.

Foram aferidas as freqüências cardíaca (FC) e respiratória (FR) nos momentos anterior a administração da clorpromazina, 20 minutos após administração da clorpromazina, ou seja, no momento anterior à aplicação do propofol e da ropivacaína (T0), 20 minutos após o bloqueio do nervo alveolar mandibular com ropivacaína (T20) e a cada 20 minutos, até a volta da sensibilidade na região anestesiada.

A partir dos resultados das médias das FC e FR nos momentos analisados, aplicou-se o teste de Dunnet $(\mathrm{P}<0,05)$ para comparar as médias dos diversos momentos em relação ao T0.

A realização deste trabalho foi aprovada pela Comissão de Ética e Biossegurança do Programa de Pós-graduação em Medicina Veterinária, área de concentração clínica e cirurgia, da Faculdade de Medicina Veterinária da Universidade Federal de Uberlândia, onde o mesmo foi realizado obedecendo às normas éticas.

\section{RESULTADOS E DISCUSSÃO}

Com a dissecação das mandíbulas dos três gatos, pode-se determinar o local de aplicação do anestésico, no qual se observou uma distância de $1,0 \mathrm{~cm}$ rostral ao processo angular e $0,5 \mathrm{~cm}$ dorsal à borda ventral do ramo da mandíbula, conforme utilizaram GROSS et al. (2000).

Neste trabalho, optou-se pela dose de 1,0mg $\mathrm{kg}^{-1}$ de ropivacaína, como recomendada por MUIR III et al. (2001) para anestesias regionais, observando efetividade no bloqueio. A concentração utilizada foi de $0,5 \%$, permitindo uso de um volume satisfatório do anestésico, sendo que não houve falhas no bloqueio como citam POTOCNIK \& BAJROVIC (1999).

Na amostra analisada, observou-se variação da latência sensitiva entre 12 e 38 minutos $(22 \pm 6,82)$ e 
duração do bloqueio entre 90 e 200 minutos (164,25 \pm 32,69). Entretanto, GROSS et al. (2000), ao utilizarem cloroprocaína para bloqueio do nervo alveolar mandibular de gatos, encontraram período de latência de 10 minutos com duração de 90 minutos. Este maior tempo de duração da anestesia observado neste estudo foi devido ao fato de a ropivacaína promover bloqueio de longa duração, três vezes mais potente que a cloroprocaína. A latência de 22 26,82 minutos encontrada no estudo deveu-se ao fato de a ropivacaína ser um anestésico de início de ação intermediário (MUIR III et al., 2001).

Através do diagrama de dispersão (Figura 1), pode-se observar a não correlação entre a latência sensitiva e a duração do bloqueio. Estes dados estão de acordo com DELFINO et al. (1999) que comentam que este fármaco possui um período de latência elevado, estando este relacionado a uma menor absorção a partir do local de injeção devido à sua forma levógira. Sendo assim, o período de latência não tem correlação com a duração do bloqueio (RAMOS et al., 2000).

Quando se aplicou o teste de Dunnet para os valores de FC (Tabela 1) comparando T0 (163 \pm 31$)$ com os demais momentos observou-se diferença

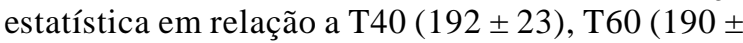
22), T80 (186 \pm 26$)$, T100 (187 \pm 15$),$ T120 (188 \pm 16$)$ e T140 (187 \pm 22$)$. Isto se deveu ao fato de os animais, no momento T0, estarem sob efeito da clorpromazina que bloqueia, no sistema nervoso central, neurotransmissores como a serotonina e a dopamina e, também causa depressão do sistema reticular. Promoveu-se assim, diminuição da FC em T0 devido a tranqüilização do paciente (MUIR III et al., 2001; FANTONI \& CORTOPASSI, 2002).
O mesmo teste foi realizado para FR, comparando os diversos momentos (Tabela 1) com T0 (46 \pm 15$)$. Foi observada diferença estatística em relação aos momentos T80 (37 \pm 10$)$, T100 (36 \pm 9), T120 (38 \pm $6)$, T160 (36 \pm 5$)$, T200 (27 \pm 3$)$ em que se notou uma redução gradativa desta. Esses resultados provavelmente sejam resultados do efeito dos fármacos clorpromazina e propofol sobre o centro respiratório e pelo relaxamento muscular induzido por estes, em que se incluem os músculos da respiração (SOUZA et al., 2002).

O bloqueio do nervo alveolar mandibular proporcionou anestesia da região dos dentes molares, pré-molares, caninos, incisivos, pele e mucosa da bochecha e lábio inferior da face direita da mandíbula. Fatos esses ocorridos devido ao nervo alveolar mandibular ser sensorial, e por sua divisão após penetrar no canal mandibular, fornecendo ramos para os dentes molares, caninos e incisivos, assim como pela emersão do nervo mentoniano, através do forame mentoniano, o qual inerva a região mentoniana (GODINHO \& GETTY, 1986; EVANS \& LAHUNTA, 1994; SCHALLER, 1999). Em todos os animais deste experimento, obteve-se anestesia da região lateral e ventral direita das mandíbulas, diferindo dos resultados encontrados por GROSS et al. (2000), que não obtiveram bloqueio do nervo alveolar mandibular em $50 \%$ dos gatos e AFSAR et al. (1998) que descreveram falha de 29 a 35\% da anestesia do nervo alveolar nos homens. O sucesso de bloqueio em $100 \%$ dos casos pode ser explicado pela deposição do anestésico próximo ao nervo alveolar mandibular e milo-hióide, permitindo a embebição destes, sem, contudo, lesá-los.

Não foram observados sinais aparentes de intoxicação, nem hematomas, no local de aplicação deste anestésico local. Diferindo de TAKASUGI et al.
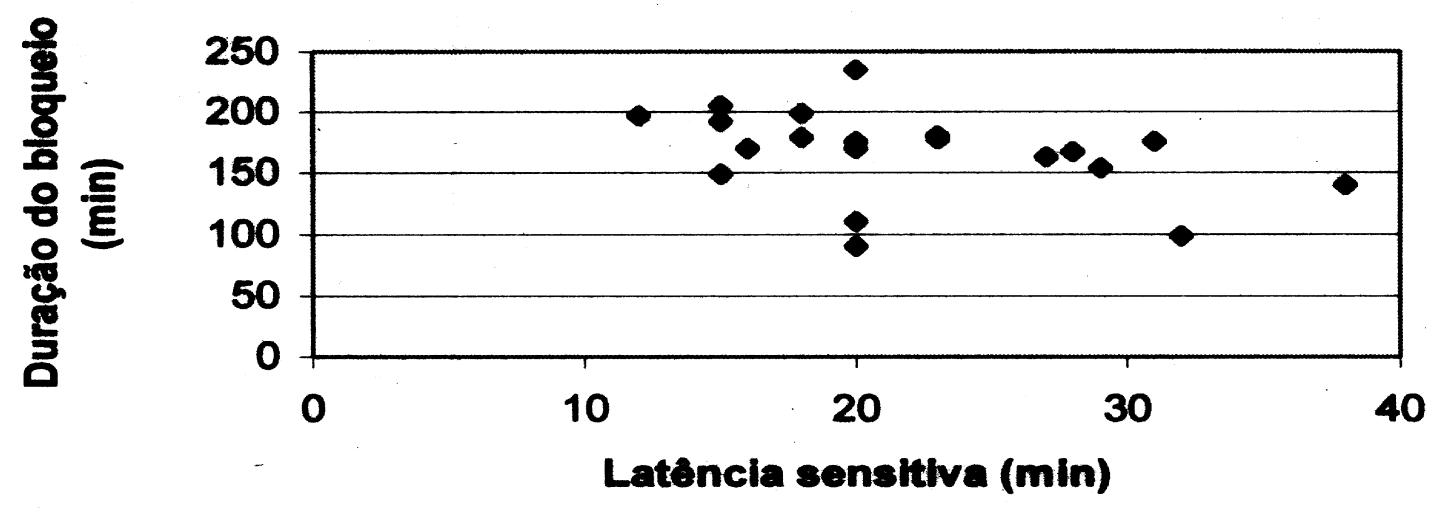

Figura 1 - diagrama de dispersão evidenciando a não correlação entre a duração do bloqueio e a latência sensitiva da anestesia do nervo alveolar inferior com ropivacaína a $0,5 \%$ em gatos.

Ciência Rural, v.36, n.2, mar-abr, 2006. 
Tabela 1 - Valores obtidos para as freqüências respiratória e cardíaca após o bloqueio do nervo alveolar mandibular com ropivacaína a 0,5\% em gatos.

\begin{tabular}{llccc}
\hline \multirow{2}{*}{ Momentos } & \multicolumn{2}{r}{ Frequência respiratória } & \multicolumn{2}{c}{ Frequência cardíaca } \\
\hline \multirow{2}{*}{ T0 } & Médias & Desvio padrão & Médias & Desvio padrão \\
T20 & 46,15 & 15,78 & 163,55 & 31,04 \\
T40 & 43,90 & 9,95 & 181,30 & 28,53 \\
T60 & 43,20 & 8,02 & 192,55 & 23,47 \\
T80 & 37,80 & 10,89 & 186,35 & 26,01 \\
T100 & 36,11 & 9,74 & 187,05 & 15,82 \\
T120 & 38,11 & 6,42 & 188,52 & 16,04 \\
T140 & 39,00 & 7,21 & 187,31 & 22,72 \\
T160 & 36,60 & 5,93 & 195,20 & 25,10 \\
T180 & 37,33 & 3,77 & 186,66 & 30,61 \\
T200 & 27,00 & 3,00 & 204,00 & 50,91 \\
\hline
\end{tabular}

(2000) que relataram intoxicação com a utilização de lidocaína a $2 \%$ com vasoconstrictor no bloqueio do nervo alveolar inferior no homem. Também não foi notado parestesia permanente do nervo alveolar mandibular, como a descrita por POGREL \& THAMBY (2000) e GIULIANI et al. (2001). Isto provavelmente ocorreu devido a eficiência da técnica utilizada, sem aparente danos aos nervos, pela manipulação da agulha estando de acordo com GROSS et al. (1997). Além disso, não ocorreu injeção intravascular que poderia causar neurotoxicidade (DELFINO et al., 1999; MARANHÃO et al., 2000; TAKASUGI et al., 2000), que também foi evitada em prol da baixa toxicidade da ropivacaína em relação à lidocaína e à bupivacaína (DELFINO et al., 1999; DELFINO et al., 2000).

\section{CONCLUSÃO}

A anestesia do nervo alveolar mandibular com ropivacaína a 0,5\% foi eficiente, de fácil aplicação, permitiu um bloqueio de duração entre 90 e 200 minutos, atingindo as regiões dos dentes pré-molares, molares, caninos, incisivos, pele e mucosa da bochecha e lábio inferior, sem causar efeitos colaterais e reações adversas em gatos sem raça definida.

\section{REFERÊNCIAS}

AFSAR, A. et al. Radiographic localization of mandibular anesthesia landmarks. Oral Surgery, Oral Medicine, Oral Pathology, Saint Louis, v.86, n.2, p.234-241, 1998.

CEDIEL, R. et al. Anestesia em odontologia. In: ASCASO, F.S.R. et al. Atlas de odontologia de pequenos animais. São Paulo: Manole, 1999. Cap.6, p.97-110.
DELFINO, J. et al. Estudo comparativo entre a bupivacaína $0,5 \%$ e ropivacaína $0,5 \%$ isobáricas na anestesia subaracnóidea para cirurgia ortopédica. Revista Brasileira de Anestesiologia, Rio de Janeiro, v.49, n.3, p.160-164, 1999.

DELFINO, J. et al. Estudo comparativo entre a ropivacaína $0,5 \%$ e $0,75 \%$ isobáricas na anestesia subaracnóidea para cirurgia ortopédica. Revista Brasileira de Anestesiologia, Rio de Janeiro, v.50, n.3, p.207-211, 2000.

EVANS, H.E.; LAHUNTA, A. de. Miller - Guia para a dissecação do cão. 3.ed. Rio de janeiro: Guanabara Koogan, 1994. 206p.

FANTONI, D.T.; CORTOPASSI, S.R.G. Anestesia em cães e gatos. São Paulo: Roca, 2002. 389p.

GODINHO, H.P.; GETTY, R. Sistema nervoso periférico. In:GETTY, R. Sisson/Grossman anatomia dos animais domésticos. 5.ed. Rio de Janeiro: Guanabara Koogan, 1986. V.2, p.1583-1595.

GIULIANI, M. et al. Inferior alveolar nerve paresthesia caused by endodontic pathosis. A case report and review of the literature. Oral Surgery, Oral Medicine, Oral Pathology, Saint Louis, v.92, n.6, p.670-674, 2001.

GROSS, M.E. et al. Regional anesthesia of the infraorbital and inferior alveolar nerves during noninvasive tooth pulp stimulation in halothane-anesthetized dogs. Journal of the American Veterinary Medical Association, Schaumburg, v.211, n.11, p.1403-1405, 1997.

GROSS, M.E. et al. Regional anesthesia of the infraorbital and inferior alveolar nerves during noninvasive tooth pulp stimulation in halothane-anesthetized cats. American Journal of Veterinary Research, Chicago, v.61, n.10, p.1245-1247, 2000 .

MARANHÃO, M.V.M. et al. Injeção intravascular acidental de ropivacaína. Relato de caso. Revista Brasileira de Anestesiologia, Rio de Janeiro, v.50, n.4, p.299-301, 2000 .

MUIR III, W.W. et al. Manual de anestesia veterinária. 3.ed. São Paulo: Artmed, 2001. 432p.

POGREL, M.A.; THAMBY, S.R.I. Permanent nerve involvement resulting from inferior alveolar nerve blocks. The Journal of the American Dental Association, Chicago, v.131, n.7, p.901-907, 2000.

POTOCNIK, I.; BAJROVIC, F. Failure of inferior alveolar nerve block in endodontics. Endodontics \& Dental Traumatology, Copenhagen, v.15, p.247-251, 1999.

RAMOS, G. et al. Alcalinização da ropivacaína 0,75\% para bloqueio peridural. Revista Brasileira de Anestesiologia, Rio de Janeior, v.50, n.6, p.442-449, 2000.

SCHALLER, O. Nomenclatura anatômica veterinária ilustrada. São Paulo: Manole, 1999. 614p.

SOUZA, A.P. et al. Butorfanol na anestesia com propofol em gatas pré-tratadas com levomepromazina. Ciência Rural, Santa Maria, v.32, n.4, p.589-594, 2002.

TAKASUGI, Y. et al. Clinical evaluation of inferior alveolar nerve block by injection into the pterygomandibular space anterior to the mandibular foramen. Journal of the American Dental Society of Anesthesiology, Rego Park, v.47, p.125129, 2000. 